\title{
Saúde de Lésbicas, Gays, Bissexuais, Travestis e Transexuais: da Formação Médica à Atuação Profissional
}

\section{Health of Lesbians, Gays, Bisexuals, Transvestites and Transsexuals: from Medical Training to Professional Activities}

Flávia Rachel Nogueira de Negreiros ${ }^{I}$ Breno de Oliveira Ferreira ${ }^{I I}$ Danilo de Negreiros Freitas ${ }^{I}$ José Ivo dos Santos Pedrosa ${ }^{I I I}$ Elaine Ferreira do Nascimento ${ }^{I I}$

\section{PALAVRAS-CHAVE}

- Educação Médica.

- Atenção Básica.

- Minorias Sexuais.
I Universidade Federal do Piauí, Teresina, Piauí, Brasil.

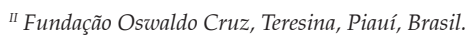

III Universidade Federal do Piauí, Parnaíba, Piauí, Brasil.

\section{RESUMO}

Introdução: A população LGBT (lésbicas, gays, bissexuais, travestis e transexuais) está inserida em um contexto peculiar com relação ao grau de vulnerabilidade à saúde, trazendo desafios para a consolidação do Sistema Único de Saúde (SUS) enquanto sistema universal, integral e equitativo. Com isso, as práticas médicas para implementação de ações direcionadas ao cuidado LGBT podem contribuir de forma substancial para a melhoria da qualidade do acesso aos serviços básicos de saúde, porém perpassam a formação e o ensino médicos. Objetivo: Analisar a formação médica para assistência à saúde da população LGBT, na perspectiva de médicos que atuam na atenção básica. Métodos: Trata-se de uma pesquisa de caráter exploratório e descritivo, de análise qualitativa, sendo considerados sujeitos-chave 14 médicos que atuam na atenção básica. Para isto, utilizou-se a entrevista semiestruturada para a coleta e o Método de Interpretação dos Sentidos para a produção dos dados. Resultados: Emergiram duas categorias, sendo que a primeira trouxe a importância da construção do saber médico-científico para a saúde LGBT, apontando as deficiências desde a formação curricular do curso de Medicina até as capacitações que deveriam ser ofertadas pelos serviços. Já a segunda categoria mostrou o delineamento das fragilidades no cotidiano do cuidado à saúde LGBT, apontando as realidades na assistência à saúde LGBT nas unidades de saúde. Conclusão: Percebe-se a urgência na divulgação e implementação da Política Nacional de Saúde LGBT como ferramenta efetiva para promover os direitos humanos entre os profissionais médicos desde a graduação até a atuação profissional. 


\section{KEY-WORDS}

- Medical Education.

- Basic Health Care.

- Sexual Minorities.

Recebido em: 19/7/2018

Aceito em: 26/7/2018

\section{ABSTRACT}

Introduction: The LGBT (Lesbian, Gay, Bisexual, Transvestite and Transsexual) population is inserted in a very specific context, in terms of their degree of vulnerability to disease, bringing challenges for the consolidation of the Sistema Único de Saúde (SUS) as a universal, integral and equitable system. Thus, medical practices for the implementation of actions aimed at the care of LGBT can contribute substantially to improving the quality of access to basic health services, but first, health practitioners must undergo medical education and training. Objective: To analyze the medical training for health care of the LGBT population from the perspective of primary care physicians. Methods: This is an exploratory, descriptive study, with qualitative analysis. The key subjects were 14 physicians who work in Primary Care. Semi-structured interviews were used for the data collection, and the Meanings Interpretation Method was used to produce the data. Results: Two categories emerged; the first highlighted the importance of the construction of medical-scientific knowledge for LGBT health, pointing out deficiencies, from the curricular training of the medical course through to the training offered by the services. The second category outlined the fragilities of daily life in the health care of LGBT, pointing out the realities in LGBT health care in the health units. Conclusion: there is an urgent need to disseminate and implement a National Health Policy for LGBT, as an effective tool for promoting human rights among medical professionals, from graduation through to professional practice.

\section{INTRODUÇÃO}

A saúde, de forma geral, sem distinção de raça, cor ou gênero, passou a ser um direito garantido a partir da Constituição Federal de 1988, como reflexo da luta do Movimento da Reforma Sanitária ${ }^{1}$. No entanto, mesmo diante dessa conquista, o acesso aos serviços de saúde ainda tem se caracterizado como excludente para alguns grupos minoritários, como a população de lésbicas, gays, bissexuais, travestis e transexuais (LGBT)2.

De acordo com o retrato histórico da saúde da população LGBT, percebe-se a importância das determinações sociais em saúde geradas segundo a orientação sexual e a identidade de gênero no processo saúde-doença-cuidado, já que podem aumentar as vulnerabilidades das pessoas e dos coletivos e, nesse contexto, das especificidades de saúde desse público ${ }^{2,3,4}$.

Contudo, diante da história da própria sexualidade, a colocação da expressão sexual nos diferentes discursos sociais e políticos, em vez de ser silenciada, foi, ao contrário, submetida a intensas incitações que trataram de pulverizar a sexualidade em regimes de saber-poder-prazer ${ }^{5}$.

Por muitas décadas, o segmento LGBT foi marginalizado, como, por exemplo, na Segunda Guerra Mundial, quando, nos EUA, lésbicas e gays foram submetidos a diversos tratamentos com a finalidade de reverter sua orientação sexual, compreendida como antinatural, incomum e anormal. Em decorrência desses e de outros fatos, houve a morte de muitos LGBT nesse período ${ }^{6}$, o que se perpetua até os dias atuais.

O percurso de despatologização da homossexualidade se iniciou apenas em 1987, na terceira revisão do manual Diagnóstico e Estatístico dos Transtornos Mentais, da Associação Psiquiátrica Americana. O grupo dos transtornos da personalidade foi desmembrado, e algumas de suas subdivisões foram retiradas, como os desvios sexuais, que eram definidos nas duas primeiras versões como homossexualidade, fetichismo, pedofilia, travestismo, exibicionismo, sadismo, masoquismo e outros. Em 1993, a Organização Mundial de Saúde (OMS), sensibilizada com essa causa, retirou a homossexualidade do Código Internacional de Doenças (CID) 107,8.

Posteriormente, no Brasil, o Conselho Federal de Psicologia, na resolução $n^{\circ}$ 001, de 22 de março de 1999, passou a proibir os psicólogos de realizarem atendimentos às pessoas LGBT com a finalidade de reverter a homossexualidade. A separação entre orientação sexual e patologia rege o posicionamento do Conselho Federal de Medicina desde 1985, quando retirou a homossexualidade da condição de desvio sexual ${ }^{4,9}$.

No campo das políticas públicas, um importante marco foi a criação, em 2004, do Brasil sem Homofobia - Programa de Combate à Violência e à Discriminação contra LGBT e de Promoção da Cidadania Homossexual. Foi instituído pelo Conse- 
lho Nacional de Combate à Discriminação e teve ampla participação da sociedade. Foi um dispositivo disparador da busca pela integralidade da saúde dessa população, estabelecendo diretrizes para o combate à violência e à discriminação, além do respeito às especificidades e da promoção da cidadania ${ }^{10}$.

Recentemente, o Ministério da Saúde (MS) instituiu, por meio da Portaria no 2.836, de $1^{\circ}$ de dezembro de 2011, a Política Nacional de Saúde Integral de Lésbicas, Gays, Bissexuais, Travestis e Transexuais. Em paralelo, a Resolução n 2, de 6 de dezembro de 2011, estabeleceu estratégias e ações que orientam o Plano Operativo de Saúde Integral LGBT, considerado um divisor de águas para as políticas públicas de saúde no Brasil, com foco na população LGBT, e um evento histórico de reconhecimento das demandas $\mathrm{LGBT}^{9,11}$.

É conveniente destacar que as políticas de saúde voltadas para essa população foram construídas e estimuladas de forma compartilhada com os movimentos sociais. Esse fato trouxe empoderamento a tal questão, já que, mesmo com todos os avanços advindos na área da saúde, o preconceito nos estabelecimentos de saúde e a invisibilidade entre os profissionais ocultaram ainda mais essa população, fazendo dos espaços para promover saúde lugares incapazes de atender às reais necessidades da comunidade LGBT ${ }^{4,12}$.

Em paralelo aos desafios para efetivar os direitos adquiridos pela população LGBT, é importante destacar a importância de incluir na formação de profissionais médicos aspectos não meramente biológicos acerca da sexualidade humana e do cuidado em saúde com as minorias sexuais, seja ainda na graduação ou no percurso profissional nos serviços de saúde ${ }^{13}$.

Desta forma, destaca-se a relevância deste estudo em função de ser um tema amplamente discutido no âmbito das questões sociais e das ações de saúde, bem como por abordar as lacunas da formação médica em saúde LGBT. Sua importância se amplia ao considerarmos a demanda crescente por profissionais com formação adequada para assistir a população LGBT, aliada à carência de profissionais com tal capacitação.

A fim de nortear essa investigação, a situação-problema elegida consiste em relacionar as necessidades de saúde da população LGBT e a formação médica, questionando-se: Qual a percepção do médico que atua na atenção básica sobre a sua formação profissional para o cuidado à saúde da população LGBT?

Essa questão remete a um atendimento integral, equânime e humanizado. Com base nessa assertiva, levando-se em consideração a temática abordada e para obter respostas a esse problema, buscou-se analisar a formação médica para assistência à saúde da população LGBT na perspectiva de médicos que atuam na atenção básica.

\section{MÉTODO}

Trata-se de uma pesquisa exploratória e descritiva, de abordagem qualitativa, realizada numa capital da Região Nordeste, a qual apresenta índices elevados de LGBTfobia ${ }^{14}$. O estudo foi realizado em sete Unidades Básicas de Saúde (UBS), tendo como sujeitos-chave 14 médicos vinculados às unidades. Estas foram selecionadas por meio de sorteio simples e inseridas de acordo com os seguintes critérios: equipe mínima completa, cadastro atualizado no Sistema de Cadastro Nacional de Estabelecimentos de Saúde e equipe da UBS que contasse há pelo menos um ano com a mesma composição de profissionais.

Utilizou-se como critério de inclusão para os sujeitos-chave do estudo: médico com carga horária semanal de, no mínimo, 20 horas e que atue há mais de um ano na atenção básica. Foram excluídos aqueles que se encontravam de licença-saúde durante a coleta dos dados.

No contexto das técnicas e métodos para a coleta de dados, foram utilizadas entrevistas semiestruturadas, individuais, gravadas, partindo de perguntas condutoras elaboradas segundo os objetivos da pesquisa, envolvendo trajetória de formação, preparo para o atendimento à população LGBT e questões sobre a importância do cuidado, da assistência e do cenário de prática para o desenvolvimento de conhecimentos e de habilidades em saúde.

A sessão dos resultados apresenta as narrativas. Para preservar o anonimato, foi acrescido " $\mathrm{M}$ ", que representa médico, e um número arábico, que indica a sequência da entrevista realizada: (M1), (M2), (M3), (M4).

Os dados produzidos foram transcritos integralmente e analisados pelo Método de Interpretação de Sentidos ${ }^{15}$. Esse método se baseia em princípios hermenêutico-dialéticos que buscam interpretar o contexto, as razões e as lógicas de falas, ações e inter-relações entre os sujeitos. Na trajetória analítico-interpretativa dos textos, foram percorridos os seguintes passos: (a) leitura compreensiva, com vistas à impregnação, à visão de conjunto e à apreensão das particularidades do material da pesquisa; (b) identificação e recorte temático; (c) identificação e problematização das ideias explícitas e implícitas nos discursos; (d) busca de sentidos mais amplos (socioculturais), subjacentes às falas dos sujeitos da pesquisa; (e) diálogo entre as ideias problematizadas, informações provenientes de outros estudos acerca do assunto; (f) elaboração de síntese interpretativa, procurando articular o objetivo do estudo, a base teórica adotada e os dados empíricos ${ }^{15}$.

Destaca-se que o estudo também se insere na pesquisa multicêntrica denominada "Análise do acesso e da qualidade da atenção integral à saúde da população LGBT no SUS" 
(2015-2018) ${ }^{16}$, coordenada pela Universidade de Brasília e financiada pelo Ministério da Saúde.

O desenvolvimento do estudo atendeu às normas nacionais e internacionais de ética em pesquisa envolvendo seres humanos, conforme o Comitê de Ética em Pesquisa, sob o parecer de $\mathrm{n}^{\mathrm{o}}$ 1.635.158.

\section{RESULTADOS E DISCUSSÃO}

Dos 14 médicos que participaram da pesquisa, nove eram do sexo masculino e cinco do sexo feminino, e a média de idade foi 31 anos. Todos atuavam havia mais de um ano na atenção básica, e a média de tempo foi de cinco anos de serviço prestado. O compilado da análise das entrevistas fez surgir eixos principais, apresentados em duas categorias: "construção do saber médico-científico para a saúde LGBT" e "delineando fragilidades no cotidiano do cuidado à saúde LGBT".

\section{Construção do saber médico-científico para a saúde LGBT}

As Diretrizes Curriculares Nacionais do curso de Medicina e o projeto pedagógico devem orientar o currículo do curso de graduação para estabelecer critérios para o perfil acadêmico e profissional do egresso. Esse currículo deverá contribuir para a compreensão do contexto plural e da diversidade cultural, incluir dimensões éticas e humanísticas, desenvolvendo médicos com atitudes e valores orientados para a cidadania e capazes de levar à compreensão das determinações sociais, culturais, comportamentais, psicológicas, nos níveis individual e coletivo do processo saúde-doença-cuidado ${ }^{17}$.

Quando tal formação durante a graduação não contempla essas especificidades pluralistas, os cursos de pós-graduação podem ser utilizados como uma proposta de ampliação das lacunas na formação, bem como os cursos de capacitação, seminários e congressos, entre outros eventos de formação extramuros $^{18}$.

Ressalte-se que, para os conteúdos referentes à saúde LGBT, o Ministério da Saúde lançou em 2015 o curso online e gratuito da Política Nacional de Saúde Integral LGBT, desenvolvido de forma intersetorial, graças a uma parceria entre as Secretarias de Gestão Estratégica e Participativa (SGEP), Secretaria de Gestão do Trabalho e Educação na Saúde (SGETS), UNA-SUS e Universidade do Estado do Rio de Janeiro, com a colaboração do Comitê Técnico de Saúde LGBT. Com isso, os participantes do estudo foram analisados quanto aos cursos de pós-graduação e aos demais cursos de capacitação realizados sobre a temática (Quadro 1).

Entre as especialidades que constam no Quadro 1, a que mais aproxima os médicos do contexto da Saúde LGBT é a especialização em Saúde Pública, por abordar temas como hu-
QUADRO 1

Nível de formação dos profissionais que participaram da pesquisa

\begin{tabular}{lll} 
Profissional & Nível de formação & $\begin{array}{l}\text { Capacitações para } \\
\text { a saúde LGBT }\end{array}$ \\
M1 & Especialização em Saúde Pública & Não realizou \\
M2 & Residência médica em Pediatria & Não realizou \\
M3 & Especialização em Cardiologia & Não realizou \\
M4 & Especialização em Radiologia & Não realizou \\
M5 & Graduação & Não realizou \\
M6 & Residência médica em Clínica & Não realizou \\
M7 & Médica & \\
M8 & Residência médica em Pediatria & Não realizou \\
M9 & Especialização em Dor & Não realizou \\
M10 & Graduação & Não realizou \\
M11 & Residência médica em Cirurgia & Não realizou \\
\hline M12 & Intensiva & Não realizou \\
M13 & Especialização em Saúde Pública & Não realizou \\
M14 & Especialização em MBA & Não realizou \\
\hline
\end{tabular}

Fonte: Dados colhidos pelos pesquisadores

manização, determinação social em saúde e o processo saúde-doença. Vale destacar que nos anos 1990 a natureza social dessa especialização afastou o interesse dos médicos em virtude do pouco domínio dela no mercado de trabalho e por ser um segmento caracterizado como multiprofissional ${ }^{19}$, o que sofre influência até os dias atuais.

Entre os entrevistados, somente dois apresentaram especialização em Saúde Pública, aspecto que poderia colocá-los em situação mais próxima da saúde LGBT. M1 e M12, no entanto, especificaram que os conteúdos da pós-graduação não contribuíram para atender às especificidades das pessoas LGBT.

Eu nem ouvi falar sobre essa sigla (LGBT) no curso. (M1)

Eu não vou negar que não teve nenhuma discussão de humanização com os gays na especialização, e olha que fiz por uma boa instituição. (M2)

Os médicos participantes declararam também que não realizaram capacitação ou curso sobre a saúde LGBT, tampouco participaram de palestras, simpósios e seminários sobre a temática. Justificam essa deficiência por falta de oferta, insuficiência do tempo e pouco estímulo do empregador, o que 
corrobora os achados de outro estudo que também investigou a percepção médica sobre a saúde $\mathrm{LGBT}^{14}$.

Quando analisamos o ensino da sexualidade nos cursos médicos, fica claro que a qualidade da assistência à saúde sexual depende também de uma formação que envolva todos os aspectos relacionados à sexualidade, de forma ampla e não meramente biologicista ${ }^{3,14}$.

Quando se buscou conhecer a percepção do médico acerca da trajetória de formação para atendimento à população LGBT, todos os entrevistados destacaram que, durante sua formação acadêmica, não houve nenhuma disciplina com conteúdos curriculares voltados ao tema.

Quando estava na graduação, lembro que teve um seminário sobre sexualidade, que tratava das DST, formas de contágio e prevenção; foi na disciplina de Infectologia, mas tudo foi muito superficial. (M10)

Vimos um pouco na Psicologia Médica, no início do curso, e na Psiquiatria também, quando o mesmo professor tratou dos transtornos sexuais, de forma rápida. A gente estava preocupado mesmo era com os casos clínicos, as doenças. (M8)

Os entrevistados referiram questões generalizadas abordadas a partir de temáticas relacionadas à sexualidade em seu contexto simplista em disciplinas como Psiquiatria, Infectologia, Urologia e Psicologia Médica, com inserções avaliadas como insuficientes. Os dados apresentados por este estudo se assemelham aos encontrados numa pesquisa ${ }^{13}$ em que os temas mais discutidos em relação à sexualidade e a outras particularidades foram relacionados ao câncer, ao aborto, às IST e ao HIV/aids. Em assuntos que enfocaram questões relacionadas à sexualidade, as abordagens enfatizavam prioritariamente as disfunções sexuais, com menor evidência para a homossexualidade e os direitos sexuais reprodutivos ${ }^{13}$.

Por conseguinte, destaca-se que a formação dos entrevistados não se distancia da realidade nacional. No Brasil, apesar da existência de uma política pública voltada ao atendimento integral e humanizado da população LGBT e das propostas de reformulação das estruturas curriculares dos cursos de graduação em Medicina a partir das novas diretrizes curriculares, ainda existem deficiências do cuidado à saúde na atenção básica, reflexo das fragilidades no campo acadêmico, seja na graduação, em especializações, residências ou capacitações profissionais $^{14}$.

A atuação médica em questões que envolvem a saúde sexual se mostra limitada no que diz respeito à saúde geral ${ }^{13,14}$, conforme esboça um dos médicos entrevistados:
Eu trabalho aqui há quatro anos e nunca me chamaram para nenhum curso, nenhuma capacitação, nada! Eu não tenho como saber de política, direitos, de necessidades assim, do nada. (M7)

Acredita-se que o processo de mudança de concepção com relação à população LGBT não repousa somente na melhoria do atendimento nas unidades de saúde, mas, principalmente, no modo de pensar e agir dos profissionais de saúde envolvidos nesse processo. Ressalte-se que o modelo heteronormativo influencia na atenção à saúde LGBT, e um dos cernes dessa mudança de paradigma está no ensino, na compreensão das especificidades da população LGBT desde a formação nas escolas médicas ${ }^{20}$.

Existem inquietudes com o modelo de ensino ofertado nas escolas médicas, onde as propostas educacionais, por vezes, ainda privilegiam uma concepção tecnicista, voltada à valorização de metodologias de ensino centradas no produzir, e não no questionar. Essas mudanças reproduzem experiências já vivenciadas, sem uma reflexão crítica acerca da sociedade moderna e das demandas sociais. Além disso, essas modificações curriculares tendem a copiar modelos de ensino de outras nações, que não atendem às reais necessidades da população que será assistida ${ }^{13,20,21}$.

\section{Delineando fragilidades no cotidiano do cuidado à saúde LGBT}

A qualidade do atendimento médico ofertado à população LGBT não é uma questão contemporânea, e os usuários LGBT podem encontrar na consulta de atendimento um profissional que encara a orientação sexual deles ou sua identidade de gênero como uma anomalia ou perversidade, levando essas pessoas a aparentar o que não são para não serem julgadas e a silenciar os verdadeiros motivos que as levaram até a consulta ${ }^{22}$. Dessa forma, observa-se que, mesmo 19 anos após os achados de uma pesquisa norte-americana, as falas dos médicos ainda associam a expressão sexual às anormalidades:

Olha, não dá para tratar o travesti como uma pessoa normal, porque é um paciente de alto risco, precisa ser acompanhado pelo especialista. (M1)

A maneira de falar requer mais cuidado com os homossexuais do que com as pessoas normais. (M9)

[...] francamente, eu acho que deve ter o mesmo [tratamento] de uma pessoa normal, fora dos problemas, dos complexos que ainda existem, esse tabu, mas eu acho que deve ser tratado normal, normal, eu ainda digo pra você normal. (M3) 
Os relatos extraídos no discurso parecem revelar uma rejeição irracional à homossexualidade. As pessoas LGBT não têm suas necessidades de saúde totalmente assistidas por consequência da LGBTfobia. Esses sujeitos têm medo de revelar sua orientação sexual ou identidade de gênero nos serviços de saúde por receio do preconceito e da discriminação ${ }^{3,23}$.

Destaca-se também, entre as narrativas dos médicos, uma forte ligação entre a população LGBT e a aids. Essa relação é associada à vulnerabilidade a que esse segmento foi exposto historicamente. $\mathrm{O}$ estigma e a discriminação deixaram essas pessoas ainda mais desamparadas no período da epidemia, tornando-as protagonistas da doença até os dias de hoje ${ }^{24}$.

Não dá pra não associar esse público às DST, como a aids mesmo. (M6)

$O$ atendimento aqui é bem completo, tem testes rápidos, exames de aids, sífilis, campanhas com preservativos. (M10)

Os homossexuais são bem preocupados com a saúde, vêm sempre aqui pedir os exames [...] o problema maior é a quantidade de parceiros, a aids [...] o negócio da prostituição, é isso que atrapalha mais mesmo. (M1)

Posto isso, as narrativas seguintes trazem outra questão relevante: a discriminação às travestis. Dos fatores que dificultam a garantia dos direitos aos serviços de saúde às travestis, destacam-se: o desconhecimento dos médicos em relação às demandas de saúde da travesti, a falta de resolutividade de questões específicas dessa categoria, o não uso do nome social, o julgamento moral e religioso, a reprovação pelos gestos, pelos olhares e pelas falas dos profissionais dos serviços de saúde $\mathrm{e}^{12,25}$.

Tudo bem do paciente ser homossexual. Cada um faz suas escolhas, não sou eu que vou julgar, não estou aqui para isso [...] só que quando eles se vestem de mulher, aí, chama atenção e as pessoas condenam. (M8)

[...] quem tem preconceito são médicos com idades mais avançadas, que são mais conservadores, não gostam desse tipo de atendimento, principalmente se for travesti. (M11)

A Carta dos Direitos dos Usuários da Saúde ${ }^{26}$ assegura a travestis e transexuais o direito à identificação por meio do seu nome social no preenchimento de fichas de cadastros, de formulários, de prontuários e de documentos congêneres, para atendimento de serviços de saúde. Contudo, alguns participantes apontaram discordância do uso:
[...] ter dois nomes pode causar confusão na UBS e na consulta. (M3)

A pessoa tem que aceitar seu nome como é! Não tem problema o nome não condizer com a aparência porque existem pessoas normais que têm nome de homem sendo mulher. Por exemplo, eu tenho uma prima que se chama Maria João. (M5)

A Carta dos Direitos dos Usuários da Saúde ${ }^{26}$, na Portaria no 1.820 , de 13 de agosto de 2009, do MS, reitera o direito do nome social:

Garantindo-lhe: I - Identificação pelo nome e sobrenome civil, devendo existir em todo documento do usuário e usuária um campo para se registrar o nome social, independente do registro civil, sendo assegurado o uso do nome de preferência, não podendo ser identificado por número, nome ou código da doença ou outras formas desrespeitosas ou preconceituosas.

Questionados sobre temas como hormonioterapia, acompanhamento clínico, psicoterápico, cirurgia de implante de silicone e de adequação corporal de sexo como demandas que podem surgir na UBS, os médicos mostraram não ter treinamento para agir com resolutividade, em alguns casos associando essas questões até a um transtorno mental:

Não existe, que eu saiba, serviço de referência e contrarreferência no município para essas pessoas, falo do posto de saúde. (M14)

Bem, nesse caso de mudança de sexo, eu encaminharia para o Núcleo de Apoio à Saúde da Família. Lá tem o psiquiatra, ele vai saber o que fazer. (M4)

Nossa, aí você me pegou! Encaminharia para o cirurgião geral, para ele realizar a adequação [...] não tenho conhecimento se essa cirurgia é feita aqui porque não trabalho em hospitais neste estado, só aqui neste posto e no estado vizinho. (M8)

Os recortes das falas expõem as condições que travestis e transexuais vivenciam quando necessitam de atendimento no SUS. Tais fragilidades apontam o motivo de busca por serviços clandestinos de saúde para mudança corporal por meio de práticas de automedicação de hormônios ou de maneira cirúrgico-caseira, que podem trazer inúmeras sequelas à saúde em geral $^{12,25}$.

A luta pelo direito à saúde LGBT tem como principal representante a Política Nacional de Saúde Integral de Lésbicas, 
Gays, Bissexuais, Travestis e Transexuais ${ }^{11}$. Esse marco, tão representativo no avanço para a promoção da saúde dessa população vulnerável, não era de conhecimento de nenhum dos médicos entrevistados. Com isso, a falta de conhecimento dos profissionais acaba culpando os usuários pela ineficiência no serviço, conforme apontam as narrativas dos médicos:

O preconceito e a discriminação vêm por parte da própria população, que já chega aqui se sentindo excluída e discriminada; sem motivo, vem com expressões agressivas com a equipe, sem nenhuma situação de discriminação e exclusão. (M2)

A fala de M2 mostra o desafio de trabalhar com uma população marginalizada nos serviços de saúde e que ainda é vitimizada pela própria equipe de saúde, que não compreende a exclusão vivenciada por esses sujeitos, que pouco considera as resistências no acesso aos serviços de saúde e que não (re) conhece em si as fragilidades para fornecer um atendimento de qualidade dentro do SUS, que é universal, integral e equânime².

Percebe-se que, mesmo que as políticas e os programas LGBT mostrem expectativas positivas, sua execução enfrenta grandes dificuldades ${ }^{3}$. Isto decorre das diversas barreiras diante de uma sociedade que determina a heterossexualidade como o padrão de normalidade e da dificuldade de compreensão dos motivos que afastam a população LGBT dos estabelecimentos de saúde e dos motivos da sua resistência ${ }^{4,12}$.

O preconceito é próprio deles, não é da equipe, de jeito nenhum! Eles já andam traumatizados. Alguns não se aceitam, outros a família abandona, não arrumam trabalho por causa do jeito deles. É um público fragilizado! (M2)

A narrativa do M2 vai contra o posicionamento da difusão técnico-científica em que o Ministério da Saúde reconhece situações produtoras de doença na saúde da população de gays, lésbicas, bissexuais, travestis e transexuais ${ }^{11}$, que enfatiza o quão multifacetados podem ser os fatores que levam à LGBTfobia.

As narrativas dos entrevistados foram semelhantes em vários aspectos, e, mesmo que eles estejam atendendo em unidades diferentes, suas concepções de acesso, de atendimento e de igualdade de direitos recaem, na maioria das apresentações, sobre posição, comportamento e postura dos usuários diante dos profissionais e da equipe de saúde, como se observa nas falas do M12 e do M10:

O acesso é igual para todos. Discriminação seria colocar um horário de atendimento só para esse público. (M12)
Eles não procuram muito a unidade de saúde [...] só vêm nos procurar mesmo quando alguma coisa dá errado, mas mesmo assim o acesso é o mesmo. (M10)

O acesso é tecido fragilmente, e o usuário tende a não se vincular aos serviços oferecidos, mesmo que sejam ofertados de maneira "igual"2. Entre as fragilidades características no acesso aos serviços de saúde está a concepção reducionista, identificada na pesquisa, de que os traços ou os gestos considerados femininos em homens ou masculinos em mulheres menos sensíveis ajudam o médico a identificar um paciente LGBT na UBS sem que o sujeito fale sobre sua orientação sexual ou identidade de gênero:

Eles não precisam nem se identificar, homens afeminados querem ser mulheres, e mulheres masculinizadas querem ser homens. Tem uns que não assumem, mas eu sei reconhecer. (M5)

Partindo desse pressuposto, como ficariam os sujeitos bissexuais? E os sujeitos que não apresentam artefatos do universo feminino e/ou do masculino? E os que não possuem trejeitos? A fala explicitada é extremamente segregadora e invisibiliza ainda mais esses sujeitos ao reduzi-los e colocá-los como indivíduos que necessariamente se travestem.

De acordo com as apresentações, o cuidado dessa população vai desde a maneira como é recepcionada nos consultórios, nas perguntas formuladas, nas ações, até os atos que envolvem exames físicos e apresentação de diagnóstico ${ }^{16,20}$. Os médicos afirmam:

\footnotetext{
Tem que ter o maior cuidado no que se diz para não magoar, ofende; caso contrário, os ânimos podem se alterar e quem passa a ser exposto a situações constrangedoras somos nós, com comentários ofensivos e maldosos sobre nossa competência e forma de atender. (M7)

Nossa profissão não permite discriminação, mas existem situações que fogem ao nosso controle e, quando se percebe, já estamos envolvidos em um ambiente totalmente diferente daquele que seria para promover a saúde. (M13)
}

As narrativas de M7 e M13 remetem a uma questão inquietante: existe uma formação insuficiente negligenciada pelos próprios médicos? Os médicos entrevistados não questionam sua qualificação, suas fragilidades pessoais, o próprio atendimento. Quase sempre, insistem em que a população LGBT é culpada pelo atendimento que recebe, levando a uma 
questão que é multifatorial, pois envolve usuário, serviço, comunidade e gestão ${ }^{18}$.

\section{CONSIDERAÇÕES FINAIS}

É um desafio implantar a política de atenção integral à saúde LGBT, levando em conta questões discursivas das políticas públicas e das Diretrizes Curriculares do Curso de Medicina, que não ordenam conteúdos que contemplem os aspectos mais expressivos da saúde LGBT, limitando-se a comentários e exemplificações como complemento de conteúdos de outras disciplinas. Dessa maneira, não se identificaram elementos na formação médica que contribuam para a promoção à saúde desse segmento populacional dentro do universo pesquisado. Por isso, percebeu-se a urgência de promulgar a Política Nacional de Saúde LGBT como ferramenta efetiva para promover os direitos humanos entre os profissionais médicos, desde a graduação até a atuação profissional.

Recomenda-se realizar mais trabalhos voltados a essa temática, a fim de fomentar a implantação da política, facilitando, assim, a compreensão, a resolução e a utilização de intervenções no cuidado prestado à população LGBT.

\section{REFERÊNCIAS}

1. Paiva CHA, Teixeira LA. Reforma sanitária e a criação do Sistema Único de Saúde: notas sobre contextos e autores. Hist. cienc. saúde-Manguinhos (Rio de Janeiro). 2014; 21(1): 15-36.

2. Albuquerque GA, et al. Access to health services by lesbian, gay, bisexual, and transgender persons: systematic literature review. BMC Int Health Hum Rights. 2016;16(2):110.

3. Lionço T. Que direito à saúde para a população GLBT? Considerando direitos humanos, sexuais e reprodutivos em busca da integralidade e da equidade.Saude soc. (São Paulo). 2008; 17(2): 11-21.

4. Laurentino ACN. Políticas públicas de saúde para população LGBT: da criação do SUS à implementação da Política Nacional de Saúde Integral de LGBT [dissertação]. Rio de Janeiro (RJ): Escola Politécnica de Saúde Joaquim Venâncio, Fundação Oswaldo Cruz; 2015.

5. Foucault M. História da Sexualidade I:A Vontade de Saber. Rio de Janeiro: Graal; 1993.

6. Louro GL. Gênero, Sexualidade e Educação: uma perspectiva pós-estruturalista. 5 ed. Petrópolis: Vozes; 2003.

7. Russo J, Venâncio ATA. Classificando as pessoas e suas perturbações: a "revolução terminológica" do DSM III. Rev. latinoam. psicopatol. fundam. (São Paulo). 2006; 9(3): 460-483.
8. Organização Mundial de Saúde. Classificação de transtornos mentais e de comportamento da CID - 10: Descrições Clínicas e Diretrizes diagnósticas. Porto Alegre, RS: Artes Médicas; 1993.

9. Cns. Conselho Nacional de Saúde. Plenário do Conselho Nacional de Saúde em sua Ducentésima Septuagésima Oitava Reunião Ordinária. Diário Oficial da União, 16 Mar 2016.

10. Conselho Nacional de Combate à Discriminação. Brasil sem homofobia: programa de combate à violência e à discriminação contra GLTB e promoção da cidadania homossexual. Brasília, DF: Ministério da Saúde; 2004.

11. Brasil. Política nacional de saúde integral de Lésbicas, Gays, Bissexuais, Travestis e Transexuais. Brasília: Ministério da Saúde, 2013.

12. Mello L, Perilo M, Braz CAde, Pedrosa C. Políticas de saúde para lésbicas, gays, bissexuais, travestis e transexuais no Brasil: em busca de universalidade, integralidade e equidade. Sexualidad, Salud y Sociedad (Rio de Janeiro). 2011; 1(9): 7-28.

13. Rufino AC, Madeiro AP, Girão MJBC. O Ensino da sexualidade nos cursos médicos: a percepção de estudantes do Piauí. Rev. bras. educ. med. (Rio de Janeiro). 2013; 37(2): 178-185.

14. Pereira EO, Ferreira BO, Amaral GS, Cardoso CV, Lorenzo CFG. Unidades Básicas de Saúde em Teresina-PI e o acesso da população LGBT: o que pensam os médicos? Tempus, actas de saúde colet (Brasília-DF). 2017; 11(1), 51-67.

15. Minayo MCS. O Desafio do Conhecimento. São Paulo: Editora Hucitec; 1999.

16. Ferreira BO, Tajra FS, Araújo ZAM, Pereira EO, Freitas, FRNN, Pedrosa JIS. Caminhos e vivências de investigação acerca da saúde da população LGBT em uma capital do nordeste brasileiro. Tempus, actas de saúde colet (Brasília-DF). 2017; 11(1), 41-49.

17. Resolução nº 3, de 20 de Junho de 2014. Institui Diretrizes Curriculares Nacionais do Curso de Graduação em Medicina e dá outras providências. Brasília, DF: Câmara de Educação Superior; 2014.

18. Ceccim RB, Feuerwerker LCM. O quadrilátero da formação para a área da saúde: ensino, gestão, atenção e controle social. Physis (Rio de Janeiro). 2004; 14(1), 41-65.

19. Belisário SA. Médico Sanitarista: As Muitas Faces de uma Ocupação [tese]. Rio de Janeiro (RJ): Escola Nacional de Saúde Pública, Fundação Oswaldo Cruz; 1993.

20. Miller, J. Creating inclusive helthcare environment for the GLBT community.The Official Newsletter of the Networker for Lesbian, Gay and Bissexual Concerns in Occupational Therapy. 2012; 9(1): 1-10. 
21. Briani MC. O ensino médico do Brasil está mudando? RevBrasEducMed (Rio de Janeiro). 2001; 25(3): 73-7.

22. Herek GM, Cogan JC, Gillis JR, Glunt EK. Correlates of internalized homophobia in a community sample of lesbians and gay men. Journal-Gay andLesbian Medical Association (Europa). 1997; 2(1): 17-25.

23. Araújo MAL de, Galvão MTG, Saraiva MMM, Albuquerque ADde. Relação usuária-profissional de saúde: experiência de uma mulher homossexual em uma unidade de saúde de referência de Fortaleza. Esc. Anna Nery (Rio de Janeiro). 2006; 10(2): 323-327.

24. Ayres JRCM. Epidemiologia e emancipação. 2a. ed. São Paulo: Hucitec; 2005.

25. Ferreira BO, Nascimento EF, Pedrosa JIS, Monte LMI. Vivências de travestis no acesso ao SUS. Physis. 2017; 27(4): 1023-1038.

26. Portaria $\mathrm{n}^{\mathrm{o}} 1.820$ de 13 de agosto de 2009. Dispõe sobre os direitos e deveres dos usuários da saúde. Brasília, DF: MS; 2009.

\section{CONTRIBUIÇÃO DOS AUTORES}

Flávia Rachel Nogueira de Negreiros, Breno de Oliveira Ferreira e Danilo de Negreiros Freitas participaram de todas as fases do artigo, desde a elaboração do projeto até a redação final, sob orientação de José Ivo dos Santos Pedrosa e Elaine Ferreira do Nascimento.

\section{CONFLITO DE INTERESSES}

Não há conflito de interesses.

\section{ENDEREÇO PARA CORRESPONDÊNCIA}

Flávia Rachel Nogueira de Negreiros, Centro de Ciências da Saúde, Campus Universitário Ministro Petrônio Portella, s/n - Ininga, Teresina - PI, 64049-550.

E-mail: flavia_rnc@hotmail.com 